\title{
A semi-compartmental model describing the pharmacokinetic-pharmacodynamic relationship
}

\section{Seunghee Ki}

Received December 9, 2019

\section{Corresponding author}

Seunghee Ki, M.D.

Department of Anesthesiology and

Pain Medicine, Inje University Busan

Paik Hospital, Inje University College

of Medicine, 75 Bokji-ro, Busanjin-

gu, Busan 47392, Korea

Tel: 82-51-898-6520

Fax: 82-51-898-4216

E-mail: emong0303@gmail.com

ORCID

https://orcid.org/0000-0002-1792-3771
Department of Anesthesiology and Pain Medicine, Inje University Busan Paik Hospital, Inje University College of Medicine, Busan, Korea

Frequently, we encounter the phenomenon of hysteresis in kinetic-dynamic modeling. The hysteresis loop in the concentration-effect curve suggests a time discrepancy caused by various pharmacokinetic and pharmacodynamic factors. To collapse the hysteresis loop and to simplify the concentration-effect relationship, several kinetic-dynamic modeling approaches including the effect compartment link model, turnover model (indirect response model), and tolerance/rebound model, have been used. The semicompartmental model is one method to describe the hysteresis of the pharmacokineticpharmacodynamic relationship. Furthermore, this semi-compartmental model differs from other models (full parametric approaches) as it does not require pharmacokinetic parameters to estimate pharmacodynamic parameters and $k_{\mathrm{e} 0}$. Therefore, we could employ a semi-compartmental approach in case it is difficult to apply the compartment model to pharmacokinetic data, as required for the pharmacodynamic analysis of inhalational anesthetics.

Keywords: Hysteresis; Pharmacodynamics; Pharmacokinetics; Pharmacology; Semicompartmental model.

\section{INTRODUCTION}

Pharmacokinetics (PK) and pharmacodynamics (PD) are the two main areas of integrative pharmacology. PK evaluates 'what the body does to the drug' (absorption, metabolism, distribution and excretion of drugs), and PD studies 'what the drug does to the body' (biological effects of drugs over time, the relationship between drug exposure and effects, mechanism of action of drugs). The primary purpose of PK and PD modeling is to discover the biophysiological key properties of the drug (affinity, efficacy, potency and specific systemic factor), and to predict the time course and extent of drug effect under normal physiological condition, as well as specific pathological conditions [1,2]. Usually, PK studies are first performed in various in vitro and in vivo environments, and then the concentration-effect/dose-effect relationship is inferred.
To identify a meaningful PK-PD relationship, it is important to fully correlate the pharmacological effect with the drug concentration of the appropriate compartment in vivo [3-5]. In this process, if hysteresis (a time lag between the measured concentration and the observed effect) is demonstrated, several PK-PD modeling approaches, such as effect compartment link model, turnover model/indirect response model, and tolerance/ rebound model, can be employed to describe it $[3,6,7]$. This paper introduces the semi-compartmental model, one method to collapse the hysteresis in the PK-PD relationship.

\section{PHARMACOKINETICS}

The PK model represents the time-concentration course of a drug at the measured site (usually in plasma or whole

This is an Open Access article distributed under the terms of the Creative Commons Attribution Non-Commercial License (http://creativecommons.org/licenses/by-nc/4.0) which permits unrestricted non-commercial use, distribution, and reproduction in any medium, provided the original work is properly cited. 
blood), or in additional hypothetical compartments which cannot be measured directly $[3,4]$. Compartmental PK models, that simplify complex organs and tissues into compartments and connecting lines, are most widely used $[8,9]$. Fig. 1 shows a three-compartment model.

\section{PHARMACODYNAMICS}

The PD model relates the drug concentration provided by the PK model to the observed drug effect $[2,3,5,7]$. The most commonly used PD model for describing the non-linear concentration-effect relationship is the sigmoid $E_{\max }$ model (the Hill equation [7,10], Eq. 1):

A



$$
E=\frac{E_{\max } \cdot C^{n}}{E C_{50}^{n}+C^{n}}
$$

where $E$ is effect, $C$ is drug concentration, $E_{\max }$ is the maximum effect, $E C_{50}$ is the concentration of the drug producing half of $E_{\max }$ and $n$ is the so-called steepness factor ( $n$ does not necessarily have a direct biological meaning, but it determines the slope of the curve. In the ordinary $E_{\max }$ model, $n$ is equal to 1). This model can be used extensively in other areas; for example, in the receptor theory, $E C_{50}$ reflects the potency of the drug in the system (the sensitivity of the organ or tissue to the drug) and $E_{\max }$ is the efficacy of the drug. If $n$ is less than 1 , the curve becomes hyperbolic, implying active metabolites or multiple receptor sites $[5,7]$. Fig. 2 presents the excitatory sigmoid $E_{\max }$ model including the baseline effect $\left(E_{0}\right)$, and the

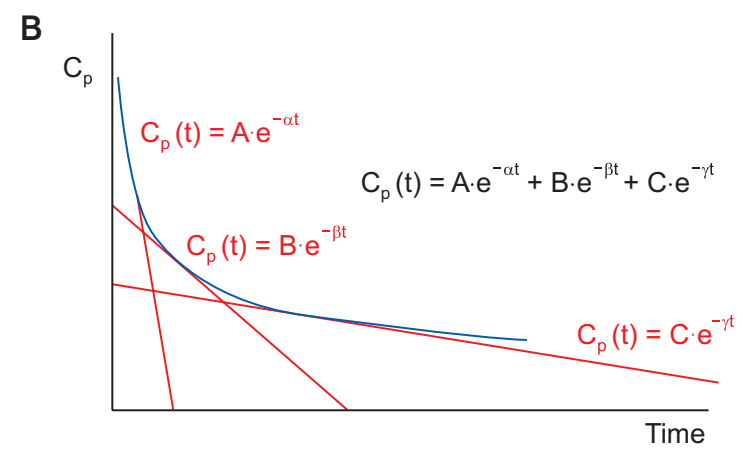

Fig. 1. Three-compartment model. (A) Scheme for three-compartment model. (B) Time-concentration curve plotted on semi-logarithmic scale in the three-compartment model following an intravenous bolus administration. $\mathrm{I}(\mathrm{t})$ : drug dose (input), $\mathrm{V}_{i}$ : distribution volume of compartment $i$, $\mathrm{k}_{i j}$ : intercompartmental fractional rate constant (micro-rate constant from compartment $i$ to compartment $j$ ), $C_{p}$ : drug concentration in plasma, A, B, C: coefficient (macro-constant), $\alpha, \beta$, $\gamma$ : exponent (macro-constant slope).

A

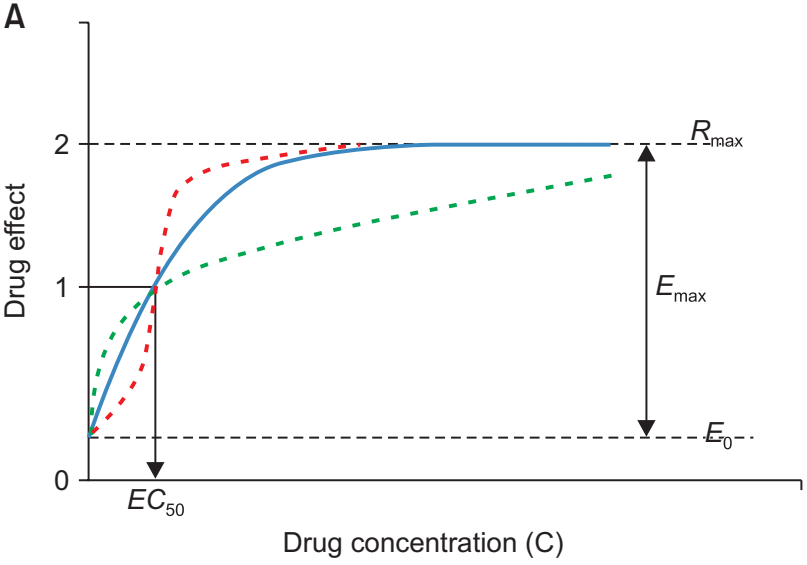

B

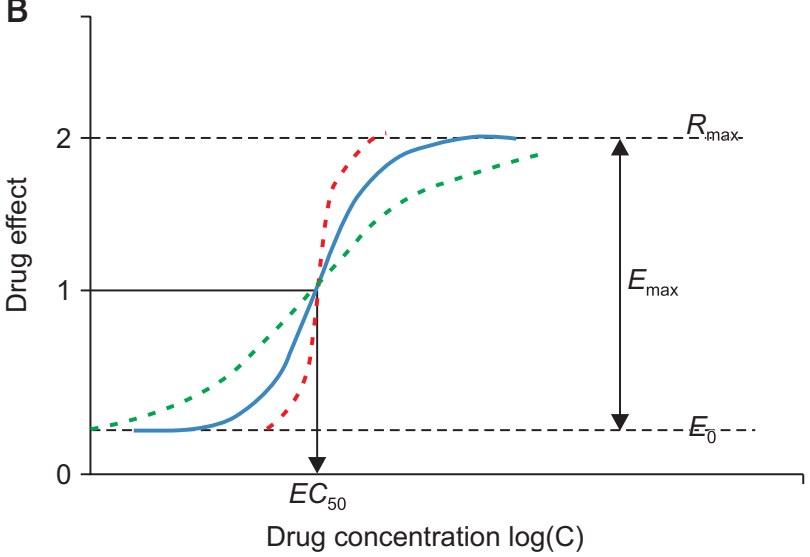

Fig. 2. The excitatory sigmoid $E_{\max }$ model with baseline $\left(E_{0}\right)$ on linear scale $(\mathrm{A})$ and semi-logarithmic scale (B); when $n$ (steepness factor) increases, the steepness of the tangent to the curve at $E C_{50}$ increases ( $n$ of the red dotted line is larger than the blue solid line, and $n$ of the green dotted line is smaller than the blue solid line). $E_{\max }$ : efficacy, the difference between baseline $\left(E_{0}\right)$ and maximum observed effect $\left(R_{\max }\right), E C_{50}$ : potency, the plasma concentration corresponding to the half of $E_{\max }$. The figure is modified from the article of Gabrielsson et al. (Pharmacodynamic concepts 2016; 199332) [7]. 
equation is functionally described as follows (Eq. 2):

$$
E=E_{0}+\frac{E_{\max } \cdot C^{n}}{E C_{50}^{n}+C^{n}}
$$

\section{KINETIC-DYNAMIC (PK-PD) MODELING}

Since PK and PD models share a common feature, i.e. concentration, they can be integrated to describe the overall dose-effect relationship, leading to useful insights into rational dose regimen design (Fig. 3) [3-5]. In the direct link model (Fig. 4A), the simplest form of the PK-PD model, the measured plasma concentration is directly linked to the effect-site concentration, as the equilibrium between both concentrations is assumed to be rapid and thus their ratio is constant under pharmacokinetic steady-state, as well as non-steadystate conditions. Hence, the measured plasma concentration can serve as an input function for the concentration-effect relationship, with the peak of plasma concentration and maximum effect occurring simultaneously $[3,5,11]$.

\section{A Direct link model}

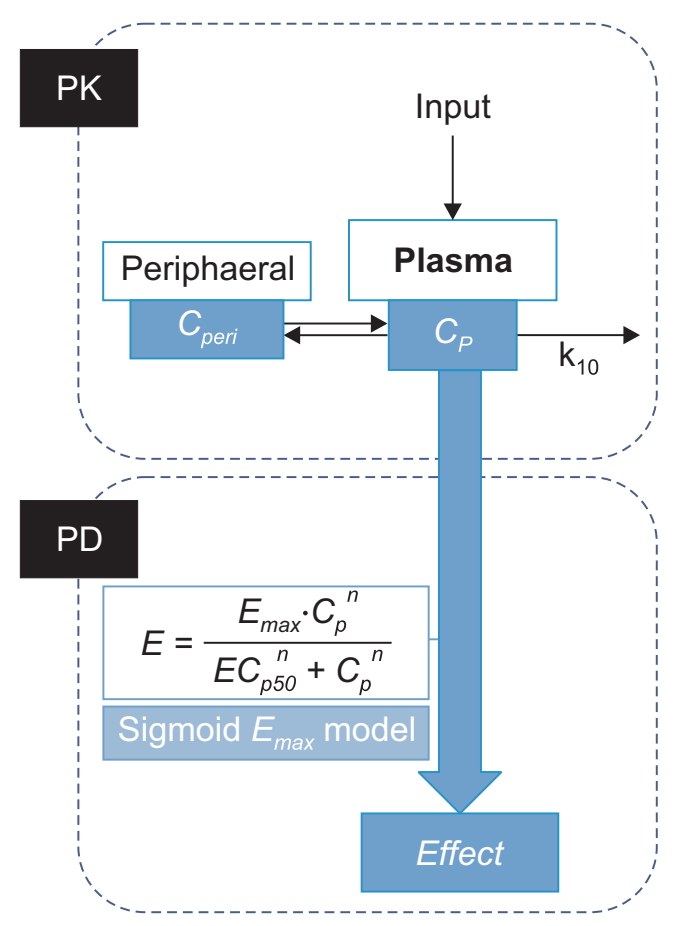

However, the assumption applied to the direct link model is often difficult to employ during actual physiological situations. In case of anesthesia induction, intravenous anesthet-

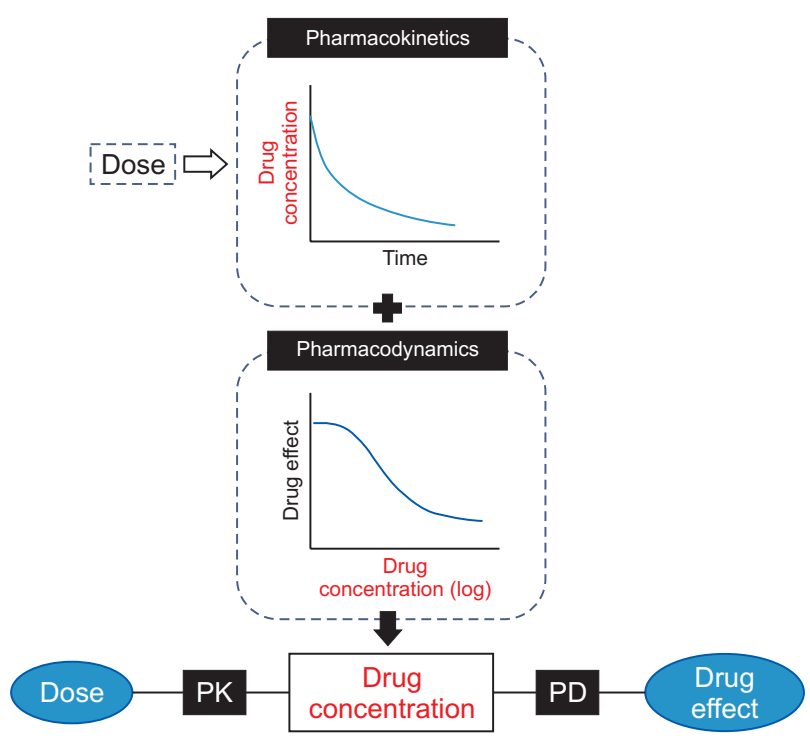

Fig. 3. Schema of presenting of the dose-effect relationship using pharmacokinetic (PK) and pharmacodynamic (PD) models.

B Effect compartment link model

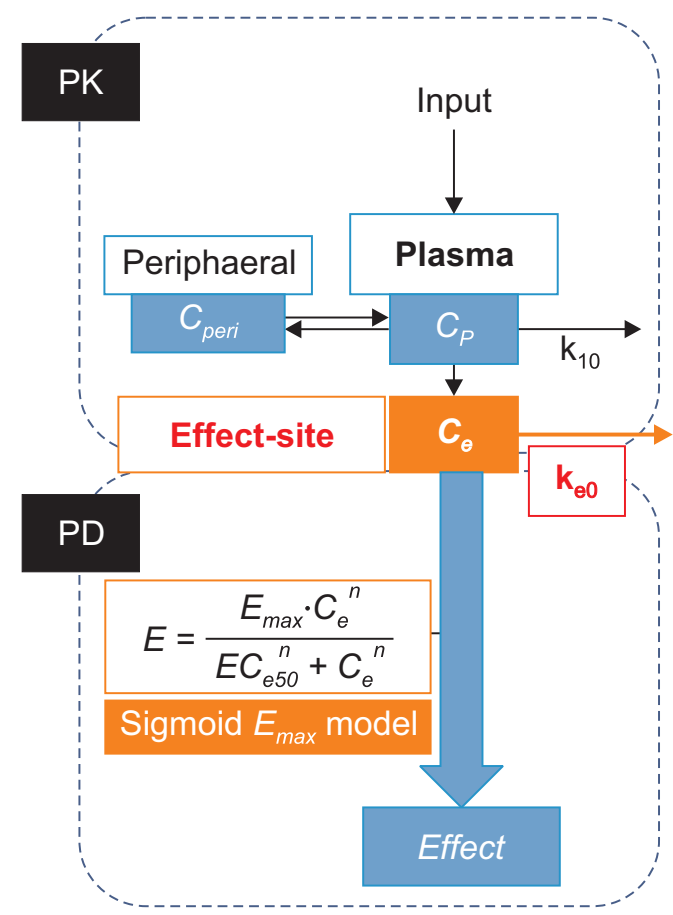

Fig. 4. Direct link model (A) vs. effect compartment link model (B). (A) In direct link model, measured plasma concentration $\left(C_{p}\right)$ and observed effect (Effect) are linked directly. (B) In the effect compartment link model, an indirect link model, $C_{p}$ and Effect are linked indirectly via a hypothetical effect site concentration $\left(C_{\mathrm{e}}\right)$. input: administrated drug dose, $k_{10}, k_{\mathrm{e} o}$ : first- order rate constants, $C_{p}$ : measured plasma concentration, $C_{\mathrm{e}}$ : effect site concentration, Effect $(E)$ : observed effect, the effect is mediated by an arbitrarily chosen sigmoid $E_{\max }$ model. 
ics and neuromuscular blockers produce a peak in the blood in a short time; however, no tracheal intubation is concurrently performed since while the drug plasma concentration reaches its maximum, further time is required for the drug to reach its maximum at the effect site. As observed, when a distribution delay between the plasma concentration and the effect site concentration (hysteresis) appears, the plasma concentration fails to represent the effect site concentration (drug effect).

\section{WHAT IS HYSTERESIS IN THE PK-PD RELATIONSHIP?}

Hysteresis is a phenomenon that occur occasionally in PKPD modeling. Hysteresis can be easily comprehended by utilizing visual thinking. When the concentration-effect curve is drawn (Fig. 5), the hysteresis loop can be observed. If you draw a vertical line at the 'concentration' axis in Fig. 5, the line crosses the curve at 2 points, indicating two different response levels for a single drug concentration. Counter-clockwise (anti-clockwise) hysteresis implies that the observed drug effect increases over time for a given drug concentration; in clockwise hysteresis, the observed drug effect decreases over time for a given drug concentration $[4,6]$.

The hysteresis loop suggests that there is a time discrepancy in the relationship between the measured drug concentration and the observed drug effect. Notably, hysteresis can occur due to a consequence of different PK and PD mechanisms, including distribution delay, input-output rate change, tolerance, formation of active metabolites, time-

A

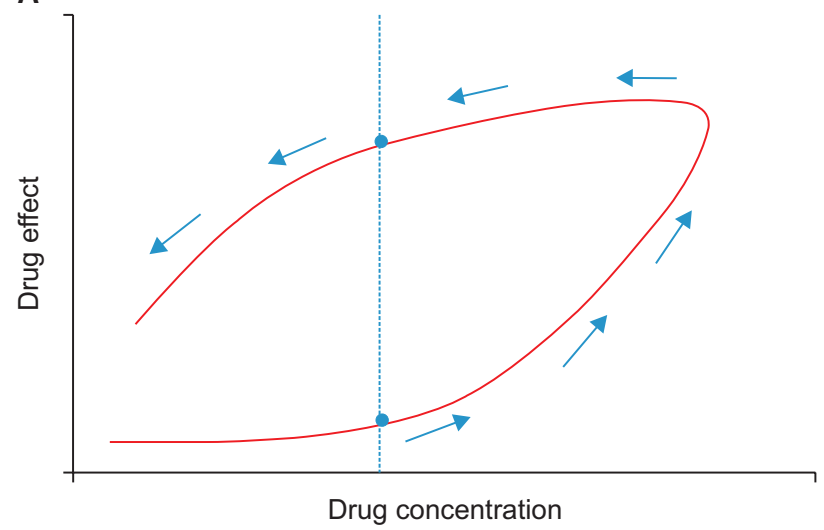

dependent protein binding, multiple receptor sites or up/ down regulation of receptor, racemic drugs, and non-stereospecific assays $[4,6]$. To collapse hysteresis and to simplify the concentration-effect relationship, several PK-PD modeling approaches, such as effect compartment link model, turnover model/indirect response model, and tolerance/rebound model, have been utilized $[3,6,7]$.

\section{EFFECT COMPARTMENT LINK MODEL}

The effect compartment link model was elaborated by Holford and Sheiner $[4,12,13]$ based on the concept of Segre [14]. This model is useful to describe the time displacement between the measured concentration and observed effect (hysteresis loop in concentration-effect curve), which often occurs due to a delayed distribution between the drug concentration in plasma and the effect site. In this model, a hypothetical



Fig. 6. Three-compartment model with an effect-compartment. The effect compartment links to the central compartment of a threecompartment model by the first-order process. I(t): drug dose (input), $\mathrm{V}_{i}$ : distribution volume of compartment $i, \mathrm{k}_{j i}$ : intercompartmental fractional rate constant (micro-rate constant from compartment $i$ to compartment $j$ ).

B

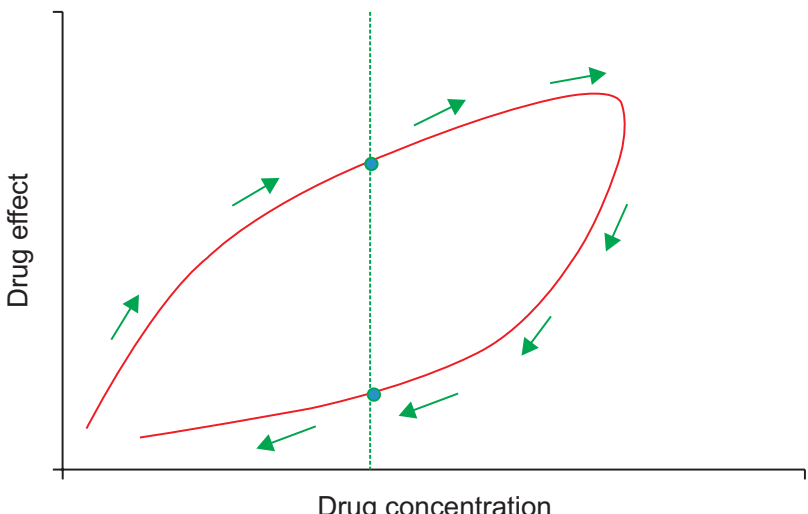

Fig. 5. Concentration-effect curve with hysteresis loop. (A) Counter-clockwise hysteresis between plasma drug concentration and observed drug effect. (B) Clockwise hysteresis between plasma drug concentration and observed drug effect. 
effect compartment is linked to the central compartment of a PK compartment model by first-order disposition kinetics (Figs. 4B, 6). It is assumed that the effect compartment has a negligible volume compared to the central compartment, receiving a negligible amount of the drug (the amount of drug influx into the central compartment from effect compartment is also negligible). The first-order rate constant $k_{e 0}$, defined as the loss of drug from the effect compartment, is not directed toward any of the PK compartments, implying excretion from the body. Therefore, $k_{e 0}$ (called 'equilibration rate constant') determines the concentration equilibrium between the plasma and effect compartment (the larger $k_{e 0}$, the faster the equilibrium, so the effect of the drug is faster) [2,3,5-7]. The relationship between the concentration at the effect compartment $\left(C_{e}\right)$ and the plasma concentration $\left(C_{p}\right)$ is expressed by the differential equation as below Eq. 3 :

$$
\frac{\mathrm{d} C_{e}}{\mathrm{~d} t}=k_{e 0} \cdot\left(C_{p}-C_{e}\right)
$$

Furthermore, the observed effect $(E)$ is correlated to $C_{e}$ by an arbitrarily chosen sigmoid $E_{\max }$ model, where $C_{e}$ is the solution to Eq. 3. The equation for the $C_{e}-E$ relationship is Eq. 4 :

$$
E=\frac{E_{\max } \cdot C_{e}^{n}}{E C_{e 50}^{n}+C_{e}^{n}}
$$

\section{SEMI-COMPARTMENTAL MODEL}

The semi-compartmental model is another estimation method that can be used to collapse hysteresis of the PKPD relationship, deriving the solution for the effect site concentration from the effect compartment link model. However, unlike the effect compartment link model in which PK parameters are first estimated and then PD parameters and $k_{e 0}$ are estimated, the semi-compartmental model does not require PK parameters (compartmental PK modeling) to estimate PD parameters and $k_{e 0}[7,15]$ (In 'NONMEM', a computer program for modeling, the effect compartment link model is named 'sequential PK-PD modeling' and the semicompartmental model is named 'direct PD fit').

There are two different solutions used in the semi-compartmental model: linear solution and log-linear solution $[7,15]$ (In 'NONMEM', a linear solution is applied when the plasma concentration is increased, and the log-linear solu- tion is applied when the plasma concentration is decreased. The control file for the semi-compartmental modeling used in 'NONMEM' is presented in the Appendix 1).

In the linear semi-compartmental solution, the plasma concentration $\left(C_{p}\right)$ obtained by assuming a piecewise linear PK model is expressed as Eq. 5.

$$
\begin{aligned}
& t_{j-1}<t \leq t_{j} \\
& C_{p}=C_{p j-1}+\lambda_{j}\left(t_{j}-t_{j-1}\right)
\end{aligned}
$$

where

$$
\lambda_{j}=\frac{C_{p j}-C_{p j-1}}{t_{j}-t_{j-1}}
$$

The integration of Eq. 3 defined the $C_{p}-C_{e}$ relationship and Eq. 5 yields the solution for concentration at the effect site $\left(C_{e}\right)$ given as Eq. 6:

$$
C_{e}=C_{e} \cdot \mathrm{e}^{-k_{00}\left(t_{j}-t_{j-1}\right)}+\frac{k_{e 0}}{k_{e 0}-\lambda_{j}} C_{p j-1}\left\{\mathrm{e}^{-\lambda_{j}\left(t_{j}-t_{j-1}\right)}-\mathrm{e}^{-k_{e 0}\left(t_{j}-t_{j-1}\right)}\right\}
$$

In the log-linear semi-compartmental solution, the plasma concentration $\left(C_{p}\right)$ is expressed in exponential form as Eq. 7, assuming a piecewise log-linear PK model.

$$
\begin{aligned}
& t_{j-1}<t \leq t_{j} \\
& C_{p}=C_{p j-1}+e^{-\lambda_{j}\left(t-t_{j-1}\right)}
\end{aligned}
$$

where

$$
\lambda_{j}=\frac{\ln C_{p j}-\ln C_{p j-1}}{t_{j}-t_{j-1}}
$$

Additionally, the log-linear semi-compartmental solution for effect site concentration $\left(C_{e}\right)$ is derived from the integration of Eq. 3 and Eq. 7, like the linear solution, and the equation is as below Eq. 8 .

$$
\begin{aligned}
C_{e}= & C_{e} \cdot \mathrm{e}^{-k_{e 0}\left(t_{j}-t_{j-1}\right)}+ \\
& \left(C_{p j-1}-\frac{\lambda_{j}}{k_{e 0}}\right)\left\{1-\mathrm{e}^{-k_{e 0}\left(t_{j}-t_{j-1}\right)}\right\}+\lambda_{j}\left(t_{j}-t_{j-1}\right)
\end{aligned}
$$

Given the observed values for $C_{p}$, an estimate of $k_{e 0}$, and starting the recursive formula with $C_{p}(0)=C_{e}=0$ (at time zero, the plasma and effect site concentrations are both zero), the time curve for $C_{e}$ can be obtained from Eq. 6 and Eq. 8. 


\section{CONCLUSION}

The semi-compartmental model allows PK-PD modeling to account for hysteresis without the PK parameters. This approach may demonstrate an advantage over the full parametric approach when model misspecification is observed in the PK model [15]. Moreover, this semi-compartmental model could be employed in studies where the measured concentration data are difficult to apply to the compartmental PK model, including the PD analysis of inhalational anesthetics using the end-tidal inhalational anesthetics concentration data (PK data) and PD data obtained by electroencephalogram based sedation depth measurements $[16,17]$.

\section{CONFLICTS OF INTEREST}

No potential conflict of interest relevant to this article was reported.

\section{REFERENCES}

1. Riviere JE, Gabrielsson J, Fink M, Mochel J. Mathematical modeling and simulation in animal health. Part I: moving beyond pharmacokinetics. J Vet Pharmacol Ther 2016; 39: 213-23.

2. Gabrielsson J, Weiner D. Pharmacokinetic and pharmacodynamic data analysis: concepts and applications. 5th ed. Stockholm, Apotekarsocieteten. 2016.

3. Derendorf H, Meibohm B. Modeling of pharmacokinetic/pharmacodynamic (PK/PD) relationships: concepts and perspectives. Pharm Res 1999; 16: 176-85.

4. Holford NH, Sheiner LB. Understanding the dose-effect relationship: clinical application of pharmacokinetic-pharmacodynamic models. Clin Pharmacokinet 1981; 6: 429-53.

5. Meibohm B, Derendorf H. Basic concepts of pharmacokinetic/ pharmacodynamic (PK/PD) modelling. Int J Clin Pharmacol
Ther 1997; 35: 401-13.

6. Louizos C, Yáñez JA, Forrest ML, Davies NM. Understanding the hysteresis loop conundrum in pharmacokinetic/pharmacodynamic relationships. J Pharm Pharm Sci 2014; 17: 34-91.

7. Gabrielsson J, Weiner D. Pharmacodynamic concepts. In: Pharmacokinetic and Pharmacodynamic Data Analysis: Concepts and Applications. 5th ed. Edited by Gabrielsson J, Weiner D: Stockholm, Apotekarsocieteten. 2016, pp 199-332.

8. Gabrielsson J, Weiner D. Pharmacokinetic concepts: multi-compartment model. In: Pharmacokinetic and Pharmacodynamic Data Analysis: Concepts and Applications. 5th ed. Edited by Gabrielsson J, Weiner D: Stockholm, Apotekarsocieteten. 2016, pp 57-72.

9. Han DW. Pharmacokinetic and pharmacodynamic modeling in anesthetic field. Anesth Pain Med 2014; 9: 77-86.

10. Hill AV. The possible effects of the aggregation of the molecules of haemoglobin on its dissociation curves. J Physiol 1910; 40: 4-7.

11. Crommelin DJA, Sindelar RD, Meibohm B. Pharmaceutical biotechnology: fundamentals and applications. 5th ed. Cham, Springer. 2019.

12. Sheiner LB, Stanski DR, Vozeh S, Miller RD, Ham J. Simultaneous modeling of pharmacokinetics and pharmacodynamics: application to d-tubocurarine. Clin Pharmacol Ther 1979; 25: 358-71.

13. Holford NH, Sheiner LB. Pharmacokinetic and pharmacodynamic modeling in vivo. Crit Rev Bioeng 1981; 5: 273-322.

14. Segre G. Kinetics of interaction between drugs and biological systems. Farmaco Sci 1968; 23: 907-18.

15. Kowalski KG, Karim A. A semicompartmental modeling approach for pharmacodynamic data assessment. J Pharmacokinet Biopharm 1995; 23: 307-22.

16. Choi BM, Koh EH, Kim MG, Kim SH, Ok SY, Noh GJ. Temporal linear mode complexity as a surrogate measure of the anesthetic drug effects during sevoflurane anesthesia. Korean J Anesthesiol 2013; 65: 385-96.

17. Bruhn J, Röpcke H, Hoeft A. Approximate entropy as an electroencephalographic measure of anesthetic drug effect during desflurane anesthesia. Anesthesiology 2000; 92: 715-26. 